\title{
Diagnosis, Treatment and Follow-up of Fetal Cardiac Arrhythmia
}

\author{
๑ Ayşe Şimşek1, (1) Tülay Demircan²
}

Iizmir Democracy University, Buca Seyfi Demirsoy Training and Research Hospital, Clinic of Pediatrics, Division of Pediatric Cardiology, İzmir, Turkey 2University of Health Sciences Turkey, Tepecik Training and Research Hospital, Clinic of Pediatrics, Division of Pediatric Cardiology, Izmir, Turkey

\begin{abstract}
Aim: The importance of managing fetal arrhythmia has increased with advances in fetal echocardiography. We aimed to describe the incidence, types, clinical characteristics, treatments, and follow-ups of patients diagnosed with fetal arrhythmia in our center.

Materials and Methods: Fetal echocardiographic examinations performed in our units between January 2016 and September 2019 were retrospectively evaluated. Fetal arrhythmias and their subtypes were identified using M-mode and Doppler echocardiography in all patients. Maternal age, gestational age, history of maternal or gestational pathology, diagnoses, and medications were recorded. Fetal arrhythmias were categorized into three main groups: 1) Irregular heart rhythm (ectopic beats), 2) Bradyarrhythmias: a ventricular rate less than 110 bpm; and 3) Tachyarrhythmias: a ventricular rate exceeding $180 \mathrm{bpm}$.

Results: A total of 60 patients were diagnosed with fetal arrhythmia, corresponding to an overall incidence of $0.5 \%$. The mean maternal and gestational age of those patients with fetal arrhythmia were $28.35 \pm 4.88$ years and $31.03 \pm 5.94$ weeks. One patient had maternal systemic disease, and four had concurrent congenital cardiac disease. Fetal tachycardia, bradycardia, and irregular heart rhythm were detected in 10 (16.6\%), 8 (13.3\%), and 42 (70\%) patients, respectively.

Conclusion: Fetal echocardiography represents the main diagnostic tool for prenatal evaluation of fetal arrhythmias, which have a variable prognosis depending on the type of arrhythmia. The most common fetal arrhythmia, the irregular heart rhythm, generally does not necessitate any treatment and resolves spontaneously. The treatment plan in patients should be based on etiology and fetal conditions.
\end{abstract}

Keywords: Fetal echocardiography, fetal arrhythmia, tachycardia, bradycardia, irregular heart rhythm

\section{Introduction}

The development of fetal echocardiography has led to an increased awareness among clinicians regarding the importance of fetal arrhythmia management (1). Although most fetal arrhythmias are of benign nature, certain types of arrhythmias may be associated with certain adverse consequences such as fetal hydrops, cardiac dysfunction, and even fetal mortality (2). Therefore, fetal heart rate (FHR) should be evaluated carefully in all pregnancies.
The detection of normal fetal cardiac rhythm is based on the documentation of regular atrial and ventricular rhythm, with a normal rate for gestational age. Each atrial activity is followed by a ventricular activity within the framework of a normal atrio-ventricular (AV) time interval, corresponding to a normal AV conduction rate of 1:1 (3).

Currently, echocardiography represents the most widely used tool for diagnosis and follow-up of fetal arrhythmias in clinical practice. Although non-invasive techniques to record electrophysiological signals from the fetal heart have been 
developed and have proven valuable in gathering important information on the pathophysiology of arrhythmias, they are mainly used as research tools and only in a limited number of centers (4).

Perinatal management of fetal arrhythmia is important to improve the outcome of an affected fetus. Accurate prenatal diagnosis is crucial for the selection of appropriate prenatal and postnatal treatments. In this study, we aimed to describe the incidence, types, clinical characteristics, treatments, and follow-ups of those patients diagnosed with fetal arrhythmia in our center.

\section{Materials and Methods}

Fetal echocardiographic examinations performed in our secondary and tertiary healthcare units between January 2016 and September 2019 were retrospectively evaluated. Fetal arrhythmias and their subtypes were diagnosed with M-mode and Doppler echocardiography in all patients. Maternal age, gestational age, history of maternal or gestational pathology, diagnoses, and medications were recorded in those patients diagnosed with fetal arrhythmia. This study was approved by the University of Health Sciences Turkey, Tepecik Training and Research Hospital Clinical Researches Ethical Committee (no: 2020/11-66).

Initially, signs of structural cardiac abnormality or cardiac failure were investigated in all fetuses. In those diagnosed with fetal arrhythmia, the type of arrhythmia was ascertained using $\mathrm{M}$-mode echocardiography and Doppler ultrasound. Simultaneous atrial and ventricular wall movements were examined via M-mode echocardiography, while the type of arrhythmia was determined using the mitral inflow and aortic outflow (mitral valve flow/aorta flow) technique via Doppler echocardiography.

\section{Classification of Fetal Arrhythmias}

Fetal arrhythmias were assessed in three main groups: 1) Irregular heart rhythm (ectopic beats); supraventricular premature beats and ventricular premature beats; 2) Bradyarrhythmias: ventricular rate less than 110 bpm (sinus bradycardia, 2:1 AV block, complete AV block), and 3) Tachyarrhythmias: Ventricular rate exceeding 180 bpm [sinus tachycardia, supraventricular tachycardia (SVT), ventricular tachycardia (VT)].

\section{Statistical Analysis}

We used the Statistical Package for Social Sciences (SPSS, version 23.0, SPSS Inc., Chicago, IL, USA). Descriptive analysis was used for demographic, clinical, and medication variables.

\section{Results}

Between January 2016 and September 2019, a total of 12,002 patients underwent fetal echocardiography. Among these, 60 were diagnosed with fetal arrhythmia. Clinical presentation included irregular rhythm in 42 patients, bradycardia in 8, and tachyarrhythmia in 10. These findings corresponded to a fetal arrhythmia incidence of $0.5 \%$ in the overall study population.

The mean maternal age in those patients with fetal arrhythmia was $28.35 \pm 4.88$ years (minimum: 19 , maximum: 38 years), while the gestational age was 31.03 \pm 5.94 weeks. One patient had maternal systemic disease, and 4 had concomitant congenital cardiac disorders. The demographic and clinical characteristics of patients with fetal arrhythmia are shown in Table I.

Ten patients, with an average maternal age of $29.2 \pm 3.64$ years and gestational age of $31.4 \pm 6.36$ weeks, had fetal tachycardia. All cases with fetal tachycardia had SVT. One patient with SVT had a cardiac anomaly consisting of a single ventricle defect and died in the first postnatal week after birth at 33 weeks of gestation.

Bradycardia was present in 8 patients, who had an average maternal age of $26.00 \pm 4.40$ years and gestational age of $27.7 \pm 5.47$ weeks. Dexamethasone treatment was started in one patient diagnosed at 29 weeks of gestation who had maternal antibody positivity. This patient was born at 38 weeks of gestation, with a heart rate between

Table I. Demographic and clinical characteristics in patients with fetal arrhythmia

\begin{tabular}{|l|l|}
\hline & $\mathbf{n = 6 0}$ \\
\hline Gestational age (weeks) (mean \pm SD) & $31.03 \pm 5.94$ \\
\hline Maternal ages (years) (mean \pm SD) & $28.35 \pm 4.88$ \\
\hline Congenital heart disease n (\%) & $4(6.6)$ \\
\hline Maternal disease $\mathrm{n}(\%)$ & $1(1.6)$ \\
\hline Mortality n (\%) & $3(5)$ \\
\hline Fetal tachycardia $\mathbf{n}(\%)$ & $10(16)$ \\
\hline Maternal ages (years) & $29.2 \pm 3.64$ \\
\hline Gestational age (weeks) & $31.4 \pm 6.36$ \\
\hline Fetal bradycardia $\mathbf{n}(\%)$ & $8(13.3)$ \\
\hline Maternal ages (years) & $26.00 \pm 4.40$ \\
\hline Gestational age (weeks) & $27.7 \pm 5.47$ \\
\hline Irregular cardiac rhythm $\mathbf{n}(\%)$ & $42(70)$ \\
\hline Maternal ages (years) & $28.28 \pm 5.23$ \\
\hline Gestational age (weeks) & $32.09 \pm 5.15$ \\
\hline SD: Standard deviation & \\
\hline
\end{tabular}


80 and $90 \mathrm{bpm}$. Another patient with left atrial isomerism and AV septal defect (AVSD) was diagnosed at 23 weeks of gestation. Although salbutamol was started, this was followed by a subsequent termination of the pregnancy. A further patient with AV block had a heart rate between 60 and $70 \mathrm{bpm}$, and was only followed up due to the absence of signs of hydrops. 4 other patients had sinus bradycardia, with a heart rate greater than $60 \mathrm{bpm}$, and these patients did not receive any medical treatment due to an absence of the signs of heart failure or hydrops. Additionally, no signs of cardiac failure were observed during their follow-up period.

Forty-two patients were found to have irregular heart rhythm. The mean maternal and gestational ages in these patients were $28.28 \pm 5.23$ years and $32.09 \pm 5.15$ weeks, respectively. Among these, only one patient had diaphragmatic herniation and supraventricular premature beats coexisting with dextrocardia. In the remaining cases, no cardiac pathology was found. The patient with diaphragmatic herniation died at postnatal 24 hours. The irregular heart rhythms were normalized in the remaining group.

Thus, the majority of our patients diagnosed with fetal arrhythmia had irregular cardiac rhythm (70\%), followed by SVT (16.6\%), and bradycardia (13.3\%). Their echocardiographic results, clinical findings, and outcomes of arrhythmia are shown in Table II.

\section{Discussion}

Fetal arrhythmias occur in a small percentage (0.6$2.0 \%$ ) of all pregnancies $(5,6)$, and the frequency of pediatric cardiologic referral of these patients is reported to be between $10 \%$ and $20 \%$ (7). Incidence rates have not been provided in some previous studies due to the admission of patients specifically diagnosed with fetal arrhythmia to tertiary referral centers (8). However, we chose to present such data since patients from a secondary healthcare facility were also included in the sample population, in addition to a tertiary center. Thus, based on our results, the incidence of fetal arrhythmia was in the order of $0.5 \%$.

\section{Fetal Tachycardia}

Tachycardia is generally defined as the presence of sustained FHR exceeding $180 \mathrm{bpm}$. The detection of a fast heart rate greater than $180 \mathrm{bpm}$ in a fetus constitutes a medical emergency since it may lead to fetal hydrops or premature delivery as well as increased perinatal morbidity and mortality. Sustained tachycardia with high ventricular rate in the early gestational weeks is more likely to be associated hydrops with fetalis. In the current study, two patients had signs of hydrops fetalis due to SVT occurring at 22 and 25 weeks of gestation.

The causes of fetal tachycardia include sinus tachycardia, re-entrant SVT, atrial flutter, atrial ectopic tachycardia, junctional ectopic tachycardia, and VT. Among these, re-entrant SVT represents the most common type of fetal tachycardia (9), comprising almost two-thirds of all cases with this condition (5). Re-entrant tachycardia mostly occurs between 24 and 32 weeks of gestation. Eight of our patients with a mean gestational age of $31.4 \pm 6.36$ weeks had re-entrant tachycardia. The second most common type of tachycardia was AF, which was detected in two cases. Conversely, junctional ectopic tachycardia and VT are uncommon and were not identified in any of our patients. Approximately $2 \%$ of those patients with fetal tachycardia have been reported to have coexisting congenital cardiac disorders $(10,11)$. In another study, $15 \%$ to $18 \%$ of fetuses with fetal arrhythmia were also found to have concomitant congenital cardiac abnormalities (12). In our study, congenital cardiac disorder was present in $12.5 \%$ of those patients with SVT and in $6.6 \%$ of those with fetal arrhythmia.

In one study involving 29 cases diagnosed with fetal arrhythmia, the subtypes were fetal tachycardia, fetal bradycardia, and irregular heart rhythm in $41.4 \%, 17.2 \%$, and $41.4 \%$ of the cases respectively (13). In the current study $16.6 \%$ of those patients with fetal arrhythmia had fetal tachycardia, and the most common type was irregular heart rhythm.

Currently, there is no consensus regarding the first agent of choice for the treatment of re-entrant SVT, and the decision should be based on the condition of the fetus. For a non-hydropic fetus, the commonly used drugs are sotalol, flecainide, and digoxin, while flecainide and sotalol have been shown to be readily transferred through the placenta and therefore may be used for hydropic fetuses (14). In a study by laeggi et al. (15), flecainide was found to be slightly more effective than sotalol in hydropic fetuses. When no response is obtained with single-agent therapy, combinations may be tried, such as amiodarone/digoxin, amiodarone/flecainide, sotalol/digoxin, and sotalol/ flecainide on the basis of studies reporting their benefits $(15,16)$. Arrhythmia was terminated with digoxin in all of our patients with re-entrant SVT.

Currently, digoxin and sotalol are used to treat AF, with sotalol preferred in the presence of hydrops. Of our two cases with $A F$, one responded to digoxin, while the other was followed up with digoxin/sotalol combination therapy. 
Table II. Echocardiographic results, clinical findings, and outcome of arrhythmia by subtype

\begin{tabular}{|c|c|c|c|c|c|}
\hline $\begin{array}{l}\text { Fetal arryhtmia } \\
\text { type }\end{array}$ & Incidence & $\begin{array}{l}\text { Echocardiographic } \\
\text { findings }\end{array}$ & Clinical findings & Treatment & Outcome \\
\hline Tachycardia & $0.08 \%$ & & & & \\
\hline Case 1 (week 25) & & Hydrops fetalis & Atrial flutter & $\begin{array}{l}\text { Digoxin } \\
\text { Sotalol }\end{array}$ & $\begin{array}{l}\text { Birth: } 36^{\text {th }} \text { week recurrent episodes of } \\
\text { SVT in postnatal period } \\
\text { Cured with propranolol }\end{array}$ \\
\hline $\begin{array}{l}\text { Case } 2 \\
\text { (week 22) }\end{array}$ & & Hydrops fetalis & Re- entrant SVT & $\begin{array}{l}\text { Digoxin } \\
\text { Sotalol }\end{array}$ & $\begin{array}{l}\text { Birth: } 34 \text { week SVT disappeared in } \\
\text { postnatal period }\end{array}$ \\
\hline Case 3 & & Single ventricle defect & Atrial flutter & Digoxin & $\begin{array}{l}\text { Recurrent episodes of SVT and } \\
\text { patients died }\end{array}$ \\
\hline Case $(4,5,6,7,8,9,10)$ & & Normal & Re- entrant SVT & Digoxin & SVT disappeared \\
\hline Bradycardia & $0.06 \%$ & & & & \\
\hline Case 1 & & $\begin{array}{l}\text { Left atrial isomerism - } \\
\text { AVSD }\end{array}$ & $\begin{array}{l}\text { CAV block } \\
\text { bradycardia }\end{array}$ & Salbutamol & Patient died in fetal period \\
\hline Case 2 & & Normal & $\begin{array}{l}\text { CAV block } \\
\text { bradycardia }\end{array}$ & - & Followed in postnatal period \\
\hline Case 3 & & $\begin{array}{l}\text { Maternal Anti-Ro + } \\
\text { Normal }\end{array}$ & $\begin{array}{l}\text { CAV block } \\
\text { bradycardia }\end{array}$ & Dexamethasone & Cured with dexamethasone \\
\hline Case 4 & & Aortic arch hypoplasia & Bradycardia & - & $\begin{array}{l}\text { Bradycardia disappeared in postnatal } \\
\text { period }\end{array}$ \\
\hline Case $(5,6,7,8)$ & & Normal & Sinus bradycardia & - & Postnatal normal \\
\hline $\begin{array}{l}\text { Irregular heart } \\
\text { rhythm }\end{array}$ & $0.34 \%$ & & & - & \\
\hline Case 1 & & $\begin{array}{l}\text { Dextrocardia, diafragmatic } \\
\text { hernia }\end{array}$ & Ectopic beats & - & Patient died in postnatal period \\
\hline Case $2-42$ & & Normal & Ectopic beats & - & Normal \\
\hline
\end{tabular}

However, this latter patient died at postnatal week 1 due to a coexisting cardiac anomaly.

\section{Fetal Bradyarrhythmia}

Fetal bradycardia is defined as a regular heart rate below 100-110 bpm without taking the gestational age of the fetus into consideration (6). It may be caused by sinus bradycardia, premature atrial contractions (PAC) with block, or AV block. Transient bradycardia, generally occurring during the second trimester, is benign and does not require treatment (14).

Persistent fetal bradycardia is relatively rare. Slow heart rate may develop due to a congenital or acquired injury in the sinoatrial node. Possible causes include viral myocarditis, inflammation and fibrosis due to collagen tissue disorders, maternal treatment with $\beta$ blockers and sedatives, fetal distress, hypoxia or acidosis. Additionally, in patients with persistent sinus bradycardia, long-QT syndrome should be investigated during the postnatal period. According to Mitchell et al. (17), 40\% of long QT syndrome cases evaluated by fetal magnetocardiography were referred due to sinus bradycardia.
Nearly $50 \%$ of cases with complete AV block are associated with complex cardiac malformations (18) (left atrial isomerism, AV septal defect, corrected transposition of the great arteries) or maternal autoantibodies (associated with autoimmune diseases such as systemic lupus erythematosus) (19).

Of the three patients with complete AV block in our study, two had coexisting anomalies (one had left atrial isomerism and AVSD, and one had maternal anti-Ro antibody positivity). In this regard, it should be remembered that the combination of complete AV block and major structural heart disease carries high mortality. When hydrops is present, the mortality rate approaches 100\% (5). In this study, the pregnancy associated with fetal left atrial isomerism was terminated.

Treatment of fetal bradycardia depends on the etiology, ventricular rate, and presence or absence of cardiac failure. If the FHR is less than 55 to 60 bpm, beta-mimetics (terbutaline, salbutamol, isoprenaline) may be tried as a first line therapy $(14,20)$. These agents may increase the 
fetal ventricular rate by around $10-20 \%$ and reverse hydrops as well (21). Immune-mediated AV block may benefit from in utero treatment with fluorinated steroids, intravenous immunoglobulin, or both. Dexamethasone is believed to reduce inflammation (22). Although no consensus exists, many clinicians administer dexamethasone 4 to $8 \mathrm{mg} / \mathrm{d}$ to treat second degree AV block, recent onset AV block, or severe cardiac dysfunction and hydrops. In our study, salbutamol treatment was given during the fetal period in the patient with atrial isomerism-AVSD, while the other patient with maternal antibody positivity benefited from dexamethasone.

\section{Irregular Cardiac Rhythm}

Irregular heart rhythm, also called premature beat, premature contraction, ectopic beat or extra-systole, can originate from the atria, the AV junction, or the ventricle, and bypasses the sinus node. Irregular heart rhythms are the most common abnormal rhythms seen in clinical practice.

Fetal ectopy occurs in up to 1 to $2 \%$ of all pregnancies and has been reported to be a relatively benign condition (5). In a study by Capuruço et al. (23), fetal ectopic beats were the most common type of fetal arrhythmia with an incidence of $55.5 \%$. Similarly, this was also the most common fetal arrhythmia, occurring in $70 \%$ of our patients. In our study, $0.34 \%$ of all pregnancies were associated with irregular heart rhythm. Most irregular beats originate from the atrium (PAC), with premature ventricular contractions (PVC) being much less frequent than PAC, with an estimated prevalence ratio of 10:1 for PAC vs. PVC (24). In clinical practice, ectopic beats are mostly detected in the third trimester of pregnancy (7). Similarly, in this study, the mean gestational age of patients with irregular heart rhythm was 32.09 weeks.

The majority of fetuses evaluated for irregular rhythm have a structurally normal heart. In a recent study of 256 singleton fetuses with an irregular heart rhythm, only two $(0.8 \%)$ had a congenital heart malformation (25). In our study, only one patient among 42 cases (2.3\%) had a coexisting cardiac anomaly. In another report describing 306 fetuses with irregular rhythm, isolated extrasystoles were diagnosed in 298 and were still present in 10 (3.4\%) at delivery (26). Among our patient group, 1 (2.3\%) had extrasystoles that persisted for up to 3 days following delivery. In patients with fetal ectopic beats, the risk of developing fetal tachycardia is estimated to be between $0.5 \%$ and $1 \%$. The presence of couplets and blocked atrial bigeminy increases this risk to approximately $10 \%$. Fetal ectopic beats are generally of benign nature, most resolving spontaneously. No medical treatment is considered necessary for these patients although it is recommended that these patients should be assessed on a weekly basis with respect to SVT and VT (14).

\section{Study Limitations}

The absence of long-term follow-up findings in the postnatal period can be assessed as a limitation of our study.

\section{Conclusion}

Fetal echocardiography represents the main diagnostic tool forprenatalevaluation offetal arrhythmias. Inall patients with suspected tachyarrhythmia or bradyarrhythmia, a fetal echocardiography should be performed to evaluate cardiac structures and functions. Fetal arrhythmias may present with variable types and prognostic consequences, with no direct effects on fetal growth and development. The etiology and fetal conditions are the main determinants of the management strategy for fetal tachyarrhythmias and bradyarrhythmias.

\section{Ethics}

Ethics Committee Approval: This study was approved by the University of Health Sciences Turkey, Tepecik Training and Research Hospital Clinical Researches Ethical Committee (no: 2020/11-66).

Informed Consent: Informed consent was not obtained because this was a retrospective study.

Peer-review: Externally and internally peer-reviewed.

\section{Authorship Contributions}

Concept: A.Ş., T.D., Data Collection or Processing: T.D., Analysis or Interpretation: A.Ş., T.D., Literature Search: A.Ş., T.D., Writing: A.Ş.

Conflict of Interest: No conflict of interest was declared by the authors.

Financial Disclosure: The authors declared that this study received no financial support.

\section{References}

1. Donofrio MT, Moon-Grady Al, Hornberger LK, et al. Diagnosis and treatment of fetal cardiac disease: a scientific statement from the American Heart Association. Circulation 2014; 129:2183-242.

2. Yaksh A, van der Does LJ, Lanters EA, de Groot NM. pharmacological therapy of tachyarrhythmias during pregnancy. Arrhythm Electrophysiol Rev 2016; 5:41-4.

3. Nii M, Hamilton RM, Fenwick L, Kingdom JC, Roman KS, Jaeggi ET. Assessment of fetal atrioventricular time intervals by tissue 
Doppler and pulse Doppler echocardiography: normal values and correlation with fetal electrocardiography. Heart 2006; 92:1831-7.

4. Sonesson SE, Acharya G. Hemodynamics in fetal arrhythmia. Acta Obstet Gynecol Scand 2016; 95:697-709.

5. Wacker-Gussmann A, Strasburger JF, Cuneo BF, Wakai RT. Diagnosis and treatment of fetal arrhythmia. Am / Perinatol 2014; 31:617-28

6. Fouron JC. Fetal arrhythmias: the Saint-Justine hospital experience. Prenat Diagn 2004; 24:1068-80.

7. Hornberger LK, Sahn DJ. Rhythm abnormalities of the fetus. Heart 2007; 93:1294-300.

8. Oztunç F, Beşikçi R, Eroğlu AG, Ahunbay G. Fetal arrhythmias: diagnosis, treatment and prognosis. Anatol / Cardiol 2003; 3:211-5.

9. Jaeggi E, Öhman A. Fetal and neonatal arrhythmias. Clin Perinatol 2016; 43:99-112.

10. Oudijk MA, Visser GH, Meijboom E). Fetal tachyarrhythmia-part I: Diagnosis. Indian Pacing Electrophysiol / 2004; 4:104-13.

11. van Engelen $A D$, Weijtens $O$, Brenner II, et al. Management outcome and follow-up of fetal tachycardia. / Am Coll Cardiol $1994 ; 24: 1371-5$.

12. Moura C, Vieira A, Guimarães H, Areias JC. Perinatal arrhythmias -- diagnosis and treatment. Rev Port Cardiol 2002; 21:45-55.

13. Gozar L, Marginean C, Toganel R, Muntean I. The role of echocardiography in fetal tachyarrhythmia diagnosis. A burden for the pediatric cardiologist and a review of the literature. Med Ultrason 2017; 19:232-5

14. Yuan SM, Xu ZY. Fetal arrhythmias: prenatal evaluation and intrauterine therapeutics. Ital J Pediatr 2020; 46:21.

15. Jaeggi ET, Carvalho JS, De Groot E, et al. Comparison of transplacental treatment of fetal supraventricular tachyarrhythmias with digoxin, flecainide, and sotalol: results of a nonrandomized multicenter study. Circulation 2011; $124: 1747-54$
16. van der Heijden LB, Oudijk MA, Manten GT, ter Heide $H$, Pistorius L, Freund MW. Sotalol as first-line treatment for fetal tachycardia and neonatal follow-up. Ultrasound Obstet Gynecol 2013; 42:285-93.

17. Mitchell IL, Cuneo BF, Etheridge SP, Horigome H, Weng HY, Benson DW. Fetal heart rate predictors of long QT syndrome. Circulation 2012; 126:2688-95.

18. Jaeggi ET, Hornberger LK, Smallhorn JF, Fouron JC. Prenatal diagnosis of complete atrioventricular block associated with structural heart disease: combined experience of two tertiary care centers and review of the literature. Ultrasound Obstet Gynecol 2005; 26:16-21.

19. Lopes LM, Tavares GM, Damiano AP, et al. Perinatal outcome of fetal atrioventricular block: one-hundred-sixteen cases from a single institution. Circulation 2008; 118:1268-75.

20. Cuneo BF, Zhao H, Strasburger JF, Ovadia M, Huhta JC, Wakai RT. Atrial and ventricular rate response and patterns of heart rate acceleration during maternal-fetal terbutaline treatment of fetal complete heart block. Am J Cardiol 2007; 100:661-5.

21. Maeno $Y$, Hirose A, Kanbe T, Hori D. Fetal arrhythmia: prenatal diagnosis and perinatal management. / Obstet Gynaecol Res $2009 ; 35: 623-9$

22. Trucco SM, Jaeggi $E$, Cuneo B, et al. Use of intravenous gamma globulin and corticosteroids in the treatment of maternal autoantibody-mediated cardiomyopathy. I Am Coll Cardiol 2011; 57:715-23.

23. Capuruço CA MC, Rezende GD, Santos R. Fetal tachyarrhythmia: diagnosis,treatment and outcome. Ultrasound Obstet Gynecol 2016; 48:167-269.

24. Batra AS, Balaji S. Fetal arrhythmias: diagnosis and management. Indian Pacing Electrophysiol ) 2019; 19:104-9.

25. Saemundsson $Y$, Johansson $C$, Wenling S, Gudmundsson S. Hepatic venous Doppler in the evaluation of fetal extrasystoles. Ultrasound Obstet Gynecol 2011; 37:179-83.

26. Cuneo BF, Strasburger JF, Wakai RT, Ovadia M. Conduction system disease in fetuses evaluated for irregular cardiac rhythm. Fetal Diagn Ther 2006; 21:307-13. 